For the interaction of the two polymers

$$
\chi_{23}(\text { Flory })=m_{2} \chi_{23}(\text { Scott-Tompa })=\frac{V_{2}}{R T}\left(\delta_{2}-\delta_{3}\right)^{2}
$$

Then, one finds

$$
\chi_{23}=\frac{\left(\chi_{12}-\chi_{13}\right)^{2}}{\left(\delta_{2}+\delta_{3}-2 \delta_{1}\right)^{2}} \frac{R T}{v}
$$

where $v$ is the molar volume of the segments in the solvent molecules. In the present work, $m_{1}=1$, so that $v \sim 100$ $\mathrm{cm}^{3} / \mathrm{mol}$. We then have

$$
\chi_{23} \sim\left(\chi_{12}-\chi_{13}\right)^{2}
$$

An inspection of the $\chi$ values in Figure 1 , curves $b$ and $c$, and for other spinodals not reported here, indicates that if eq 6 is correct, closed immiscibility loops should not be generally found, but rather spinodals like curves $b$ and $c$ in Figure 1. Possibly the loops could occur in a special case with two polymers of very similar chemical nature, which would minimize $\chi_{23}$, but of different flexibilities, which would produce different free volume or equation of state contributions in $\chi_{12}$ and $\chi_{13}$.

Koningsveld and collaborators ${ }^{7}$ have studied a system which shows a closed immiscibility loop: linear polyethylene-crystallizable polypropylene-diphenyl ether. They indicate that other such systems have been found, and point out that polymer incompatbility in solution does not necessarily imply incompatibility in the absence of solvent. Since, however, the two polymer-solvent binaries phase separate at about the same temperature, one would expect the $\chi_{1 i}$ 's to be almost identical. It is possible, therefore, that this closed loop may have a different origin than that described in the present paper.

We feel then that our main result is a theoretical support for the experimental finding of Dondos and others that polymer incompatibility in solution is increased when the polymer-solvent interactions are different. Another area where the solvent effect could be important is in the "segregation" of the blocks within isolated block copolymer macromolecules, and recent work by Dondos ${ }^{10}$ may indeed show such an effect.

Acknowledgment. We are grateful to the Petroleum Research Fund, administered by the American Chemical Society, for support of our research and to the National Research Council of Canada for a scholarship to L. Z. We thank Dr. W. Brostow of the Université de Montréal for stimulating discussion and preliminary work on this problem.

(10) A. Dondos, Makromol. Chem., 147, 123 (1971),

\title{
Efficient Computer Simulation of Polymer Conformation. I. Geometric Properties of the Hard-Sphere Model
}

\author{
Steven D. Stellman* and Paul J. Gans \\ Department of Chemistry, New York University, New York, New York 10003. \\ Received November 19, 1971
}

\begin{abstract}
A system of efficient computer programs has been developed for simulating the conformations of macromolecules. The conformation of an individual polymer is defined as a point in conformation space, whose mutually orthogonal axes represent the successive dihedral angles of the backbone chain. The statistical-mechanical average of any property is obtained as the usual configuration integral over this space. A Monte Carlo method for estimating averages is used because of the impossibility of direct numerical integration. Monte Carlo corresponds to the execution of a Markoffian random walk of a representative point through the conformation space. Unlike many previous Monte Carlo studies of polymers, which sample conformation space indiscriminately, importance sampling increases efficiency because selection of new polymers is biased to reflect their Boltzmann probabilities in the canonical ensemble, leading to reduction of sampling variance and hence to greater accuracy in given computing time. The simulation is illustrated in detail. Overall running time is proportional to $n^{5 / 4}$, where $n$ is the chain length. Results are presented for a hard-sphere linear polymer of $n$ atoms, with free dihedral rotation, with $n=$ 20-298. The fraction of polymers accepted in the importance sampling scheme, $f_{\mathrm{A}}$, is fit to a Fisher-Sykes attrition relation, giving an effective attrition constant of zero. $f_{\mathrm{A}}$ is itself an upper bound to the partition function, $Q$, relative to the unrestricted walk. The mean-squared end-to-end distance and radius of gyration exhibit the expected exponential dependence, but with exponent for the radius of gyration significantly greater than that of the end-to-end distance. The $90 \%$ confidence limits calculated for both exponents did not include either $6 / 5$ or $4 / 3$, the lattice and zero-order perturbation values, respectively. A self-correcting scheme for generating coordinates free of roundoff error is given in an Appendix.
\end{abstract}

$\mathrm{T}$ he Monte Carlo method ${ }^{1}$ was firmly established by Wall and his successors ${ }^{2-10}$ as a valuable tool for investigating

* Address correspondence to this author at the Department of Biochemical Sciences, Frick Chemical Laboratory, Princeton University, Princeton, N. J, 08540.

(1) J. M. Hammersley and D. C. Handscomb, "Monte Carlo Methods," Wiley, New York, N. Y., 1964.

(2) (a) F. T. Wall, L. A. Hiller, Jr., and D. J. Wheeler, J. Chem. Phys, 22, 1036 (1954); (b) F. T. Wall, L. A. Hiller, Jr., and W. F. Atchison, ibid., 23, 913, 2314 (1955); 26, 1742 (1957).

(3) F. T. Wall and J. Mazur, Ann. N. Y. Acad. Sci, 89, 608 (1961)

(4) (a) F. T. Wall, S. Windwer, and P. J. Gans, J. Chem. Phys., 38, 2220 (1963); (b) ibid., 38, 2228 (1963). the geometric and thermodynamic properties of polymers. The majority of previous Monte Carlo studies have been confined to the exploration of extremely simple models, such as random walks on lattices. In this paper we offer a more complete model of polymeric systems which includes lattice as well as off-lattice polymers on special cases, an ideal frame-

(5) J. Mazur and F. L. McCrackin, ibid., 49, 648 (1968).

(6) E. Loftus and P. J. Gans, ibid., 49, 3828 (1968).

(7) P. J. Gans, ibid., 42, 4159 (1965), and references cited therein.

(8) C. Domb, Advan. Chem. Phys., 15, 229 (1969).

(9) J. Mazur, ibid, 15, 261 (1969).

(10) K. K. Knaell and R. A. Scott, J. Chem. Phys., 54, 566 (1971) 
work for Monte Carlo calculations and the use of importance sampling for the reduction of sampling variance.

The remainder of this paper is devoted to elaboration of the model and method and presentation of detailed geometrical results for the hard-sphere off-lattice model. Distribution function properties of this model, and temperature-dependent properties of soft-core potential models are discussed in future papers of this series.

The primary objective is the calculation of statistical-mechanical configuration integrals of the form

$$
\langle P\rangle=\frac{\int \cdots \int P \exp (-U / k T) \mathrm{d} \tau}{\int \cdots \int \exp (-U / k T) \mathrm{d} \tau}
$$

where $P$ is the value of some conformational property, such as the squared end-to-end distance, and $U$ is the molecular potential energy. $\langle P\rangle$ denotes the canonical ensemble average of the property $P$.

Numerical polymer theory depends on representation of the energy of the molecule in terms of structural parameters. Using the adiabatic treatment of Scheraga and coworkers, ${ }^{11}$ the conformational energy is decoupled from high-frequency bending and stretching modes, permitting a classical description in terms of nonbonded and rotational barrier potential energy functions, with semiempirically determined parameters. As shown by Go and Scheraga, ${ }^{12}$ when bond lengths and bond angles are held constant, the conformational partition function can be written

$$
Q=\text { (constant) } \int \cdots \int \exp (-U / k T) \mathrm{d} \tau
$$

where $T$ is the absolute temperature and $U$ is the potential energy of the conformation. The element $\mathrm{d} \tau$ represents the minimum volume element necessary for this representation and defines the subset of phase space corresponding to the "conformation space" of the molecule. With fixed bond lengths and bond angles, the only remaining degrees of freedom are the dihedral angles about successive backbone-chain bonds. For $n$ atoms in the backbone, $n-3$ dihedral angles define the relative position of every atom to every other atom (ignoring multiatomic side chains). These angles can be considered independent variables, and from them the coordinates and potential energy can be calculated using classical potential energy functions. ${ }^{13}$

The $(n-3)$-dimensional vector $\omega$, whose elements are the individual dihedral angles $\omega_{i}$, defines a single conformation; all conformations are represented by the set of all possible vectors, $\{\omega\}$. The volume element in eq 2 can be written

$$
\mathrm{d} \tau=J(q) \mathrm{d} \omega_{1} \mathrm{~d} \omega_{2} \ldots \mathrm{d} \omega_{n-3}
$$

where the range of each $\omega_{i}$ extends from 0 to $2 \pi . J(q)$ is the Jacobian for the transformation from generalized coordinates to the $\omega$ representation. As a simple numerical factor, ${ }^{14}$ it cancels out of all equations for averages in which $Q^{-1}$ appears, such as eq 1 , and hence will be ignored. Lattice calculations restrict the values of the individual $\omega_{i}$ 's to a discrete set of suitable values. For example, the tetrahedral lattice

(11) H. A. Scheraga, Advan. Phys. Org. Chem., 6, 103 (1968); Chem. Rev., 71, 195 (1971).

(12) N. Gö and H. A. Scheraga, J. Chem, Phys, 51, 4751 (1969).

(13) G. N. Ramachandran and V. Sasisekheran, "Conformations of Biopolymers," G. N. Ramachandran, Ed., Vol. 1, Academic Press, London, 1967, p 283.

(14) J. E. Mayer and M. G. Mayer, "Statistical Mechanics," Wiley, New York, N. Y., 1940, p 230. results from fixing bond angles at $109.47^{\circ}$ and taking $\omega_{i} \epsilon$ $\left(60^{\circ}, 180^{\circ}, 300^{\circ}\right)$.

For any given structural model, the choice of energy function $U(\omega)$ determines the degree to which the properties of the random walk mimic the conformational properties of a real polymer. The simplest energy function occurs when $U$ is identically zero, for which the polymer dimensions correspond exactly with the statistics of the unrestricted random walk, whose mean-squared end-to-end distance is given (for large n) by ${ }^{15,16}$

$$
\left\langle R^{2}\right\rangle=n l^{2}
$$

where $n$ is the number of bonds and $l$ the fixed bond length. This model is known as the "freely jointed chain." Setting $U(\omega) \equiv 0$, however, is an unrealistic constraint, permitting atoms to approach arbitrarily close, even freely interpenetrating each other. This unrealistic situation can be corrected by use of a potential energy function which includes the interaction of pairs of atoms not covalently bonded to each other (excluded-volume effect). The simplest such function is the hard-sphere potential

$$
\begin{array}{cl}
U=0 & d>\rho \\
U=\infty & d \leqslant \rho
\end{array}
$$

where $d$ is the distance between the two atoms, and $\rho$ is a parameter which is roughly the sum of their van der Waals radii. The total energy of the molecule is then either zero, if no overlaps exist, or is infinite if one or more overlaps exist.

With a Monte Carlo method to be described, Wall and coworkers ${ }^{2}$ established that for the hard-sphere model

$$
\left\langle R^{2}\right\rangle=a n^{b}
$$

where $a$ and $b$ are empirical constants. It is now widely accepted that $b$ takes the value $6 / 5$ for all three-dimensional lattices in the limit of large $n .^{8}$ In the study of Loftus and Gans, ${ }^{6}$ who allowed each $\omega_{i}$ to assume angles in a continuous range $\pm \beta$ about the angles 60,180 , and $300^{\circ}$, it was found that $b$ is somewhat higher than $6 / 5$ for $\beta>0$.

Wall, et al., ${ }^{2 \mathrm{a}}$ established an empirical expression for the partition function through Monte Carlo studies on tetrahedral and cubic lattices. Their result, eq 6 below, which held for $n \gtrsim 40$, was confirmed shortly afterward by Hammersley and Morton ${ }^{17}$ as the asymptotic form for lattices. It was further observed by Wall that the number of self-avoiding walks of $n$ steps successfully completed decreases exponentially with $n$

$$
W_{n}=W_{0} \exp (-\lambda n) \text { Wall attrition law }
$$

where $W_{n}$ is the number of self-avoiding walks of $n$ steps, $W_{0}$ is an extrapolated constant, and $\lambda$, the attrition constant, depends on the lattice. Fisher and Sykes ${ }^{18}$ obtained much better fit for small $n$ (though continued good fit for large $n$ ) with the expression

$$
W_{n}=B(\mu / \sigma)^{n} n^{\alpha} \text { Fisher-Sykes attrition law }
$$

where $B$ is a constant, $\mu$ is a quantity called the "connective constant," and $\sigma$ is the number of choices for a lattice walk with no self-reversals. $\mu$ and $\lambda$ are connected by the relation

$$
\mu=\sigma \exp (-\lambda)
$$

(15) S. Chandrasekhar, Ret. Mod. Phy's., 15, 3 (1943).

(16) P. J. Flory, "Principles of Polymer Chemistry," Cornell University Press, Ithaca, N. Y., 1953, Chapter 10.

(17) J. M. Hammersley and K. W. Morton, J, Roy. Stat. Soc. Part $B, 16,23$ (1954).

(18) M. E. Fisher and M. F. Sykes, Phy's, Ret, 114, 45 (1959). 


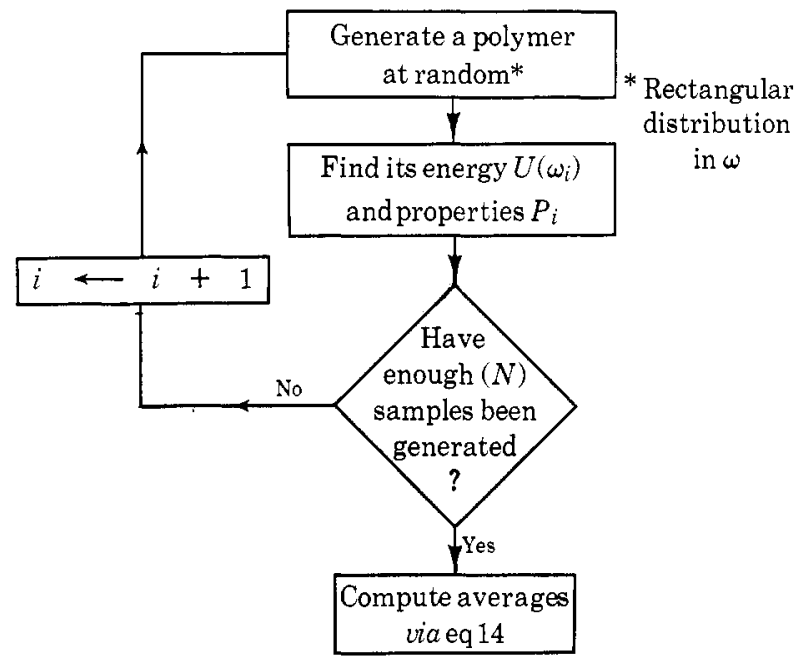

Figure 1. Sample generation by the Wall method.

so that eq 7 can be recast as an attrition law of the form

$$
W_{n}=B e^{-\lambda n} n^{\alpha}
$$

showing that the Fisher-Sykes law is a refinement of the Wall law, eq 6 , with a correction factor of $n^{\alpha}$ which loses its importance for longer walks.

For the hard-sphere energy model, the partition function $Q$ is computed as

$$
Q=N^{-1} \sum_{i=1}^{N} \exp \left(-E_{i} / k T\right)
$$

where $E_{i}$ is the energy of the $i$ th conformation and $N$ is the total number of conformations-i.e., the number of unrestricted walks. (The factor $N^{-1}$ appears because the partition function is taken relative to the unrestricted case, whose own partition function is obviously $N$.) Since a conformation has energy zero or infinity, each term contributes either a term of 1 or 0 to the sum. Thus, $Q$ is exactly equal to the number of zero-energy conformations: $Q=f_{\mathrm{A}}$, where $f_{\mathrm{A}}$ is the fraction of all unrestricted walks which are also self-avoiding. Therefore

$$
f_{\mathrm{A}}=B^{\prime} e^{-\lambda n} n^{\alpha}
$$

One of the problems encountered in computer studies of self-avoiding random walks is the difficulty and expense of investigating long chain lengths. In the sequential generation of polymer coordinates, ${ }^{2-4}$ those walks which contained one or more self-intersections were discarded without being completed and the next walk was begun. The quantitative attrition expressions, eq 6 and 7, were formulated on the basis of this observed loss of conformations. The probability of successfully extending an $n$-step walk by a single step is

$$
W_{n+1} / W_{n}=e^{-\lambda}
$$

For the tetrahedral lattice, $\lambda \approx 0.04$. With constant computer time for generating a new segment, total time for successful walks of $n$ steps increases exponentially with $n$. A great deal of effort ${ }^{7,19}$ has been expended inventing ways to combat this attrition. One of the most successful is the WallErpenbeck $s-p$ method, ${ }^{20}$ whereby self-intersecting walks are not immediately discarded, but merely restarted from a point

(19) F. T. Wall, S. Windwer, and P. J. Gans, J. Chem. Phys., 37, 1461 (1962).

(20) F. T. Wall and J. J. Erpenbeck, ibid, 30, 634 (1959).

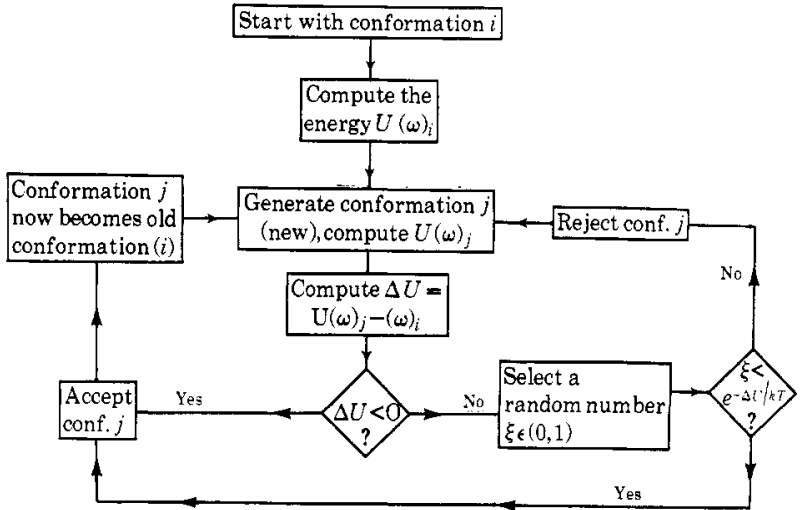

Figure 2. Flow chart of the importance sampling biasing scheme for polymer generation.

prior to the intersection; statistical biasing factors are then introduced into the results to take account of this. The computation time, however, is still exponential.

This $s-p$ method must not be confused with improved sampling methods described below. In the present work, the average properties of polymers are obtained by evaluating eq 1 with a technique known as Monte Carlo with importance sampling, ${ }^{21}$ where integration is restricted to regions of conformation space where the argument is appreciable, i.e., "important regions." In the Wall method, all regions were sampled equally. Since eq 6 implies that the "important" fraction of conformation space decreases exponentially with $n$, the majority of computing time was wasted sampling polymers of high energy which contributed negligibly to the averages.

In 1963 Fluendy ${ }^{22}$ published the first study in which a more sophisticated sampling technique (method of choosing the random vectors $\omega$ ) was used, but his investigations were restricted to alkanes below $\mathrm{C}_{12}$. More recently, $\mathrm{Lal}^{23}$ published a study in which polymers were generated on two- and threedimensional lattices by a similar procedure, and subject to a nominal soft-core potential. Their method, and ours, differs from that of Wall in the following respects.

In order to obtain a correct estimate of a property $P$ it is necessary to sample from a space characterized by the Boltzmann distribution. In the Wall method, samples (polymers) are drawn at random from a rectangular distribution and the Boltzmann factor is applied afterward. This sampling process can be represented schematically as in Figure 1.

Importance sampling, introduced by Metropolis, et al., ${ }^{24}$ in 1953 , and discussed in detail by Wood, ${ }^{21}$ operates on the principle of selecting new states with their Boltzmann probabilities, rather than totally at random, and is illustrated in Figure 2. In either case, the average statistical-mechanical properties are evaluated with following equation ${ }^{21}$

$$
\hat{P}=\hat{Q}^{-1} N^{-1} \sum_{i=1}^{N} P_{i} \exp \left(-U_{i} / k T\right)
$$

where $\hat{P}$ is the Monte Carlo estimate of $\langle P\rangle, N$ is the number of samples generated, $P_{i}$ is the value of the property for the $i$ th sample, and $U_{i}$ is its energy. Consideration of sampling

(21) W. W. Wood in "Physics of Simple Liquids," H. V. N. Temperley, J. S. Rowlinson, and G. S. Rushbrooke, Ed., North-Holland Publishing Co., Amsterdam, 1968, Chapter 5.

(22) M. A. D. Fluendy, Trans. Faraday Soc, 59, 1681 (1963).

(23) M. Lal, Mol. Phys., 17, 57 (1969).

(24) H. Metropolis, A. W. Rosenbluth, M. N. Rosenbluth, A. H. Teller, and E. Teller, J. Chem. Phys., 21, 1087 (1953). 


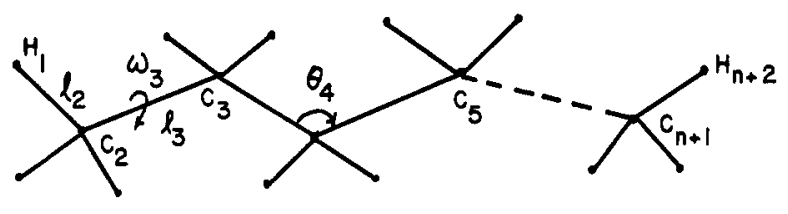

Figure 3. Definition of structural parameters for $n$-alkanes.

weights for either method results in the following expressions for any average polymeric property. In the Wall method

$$
\hat{P}=\frac{\sum P_{i} \exp \left(-U_{i} / k T\right)}{\sum \exp \left(-U_{i} / k T\right)}
$$

while in importance sampling

$$
\hat{P}=N^{-1} \sum_{i=1}^{N} P_{i}
$$

In both eq 14 and 15 , the summations extend from 1 to $N$, the number of samples generated.

In summary, with the biased sampling method described here, every sample contributes significantly, whether by its being accepted or being rejected, thereby increasing the statistical weight of the previous sample. Furthermore, the energy is continually being driven downward toward more probable regions of conformation space, while in principle any conformation, however unlikely, has a finite nonzero probability of being reached.

New states are reached with probabilities which depend only on the previous state or conformation. Hence the evaluation of the integrals in eq 1 , which is our primary goal, is seen to correspond to a Markoffian random walk in the molecular conformation space. This is considerably different from the original Brownian motion analogy in which the computer simulation was essentially an enactment of the random walk path traced by an individual particle.

\section{(I) Computational Model and Method}

A. Structural Model. The main geometric parameters of a linear alkane or polyethylene chain are depicted in Figure 3. These parameters are defined as follows: $\mathrm{C}_{i}=$ carbon atom $i ; \mathrm{H}_{i}{ }^{\prime}, \mathrm{H}_{i}{ }^{\prime \prime}=$ hydrogen atoms attached to $\mathrm{C}_{i} ; \mathrm{H}_{1}$, $\mathbf{H}_{n+2}=$ terminal hydrogen atoms; $n=$ number of carbon atoms in the chain; $l_{i}=$ distance from $\mathrm{C}_{i-1}$ to $\mathrm{C}_{i} ; \omega_{i}=\mathrm{di}$ hedral angle about $l_{i}$; and $\theta_{i}=\angle \mathrm{C}_{i-1} \mathrm{C}_{i} \mathrm{C}_{i+1}$.

The values of these parameters were chosen to correspond as closely as possible to actual values (many prior Monte Carlo studies used $\mathrm{C}-\mathrm{C}$ bond lengths of $\sqrt{3}$ for computational convenience). Their values for alkanes have been established by electron diffraction studies such as those of Kuchitsu. ${ }^{25}$ In a thorough investigation of the computational properties of these values, Scott and Scheraga ${ }^{26}$ showed that variations in these parameters within a small range did not have a serious effect on the estimated energies of various conformations, at least within experimentaly detectable limits. In particular, the $\mathrm{C}-\mathrm{C}$ bond length was chosen to be $1.54 \AA$, and the backbone bond angle was taken to be tetrahedral, in order to take advantage of certain computational conveniences in correcting for roundoff error.

For the hard-sphere model, the total energy $U_{i}$ of the $i$ th polymer generated is simply

$$
U_{i}=\sum_{j<k} f\left(r_{j k}\right)
$$

(25) K. Kuchitsu, Bull. Chem. Soc. Jap., 32, 748 (1959).

(26) R. A. Scott and H. A. Scheraga, J. Chem. Phys., 44, 3054 (1966).

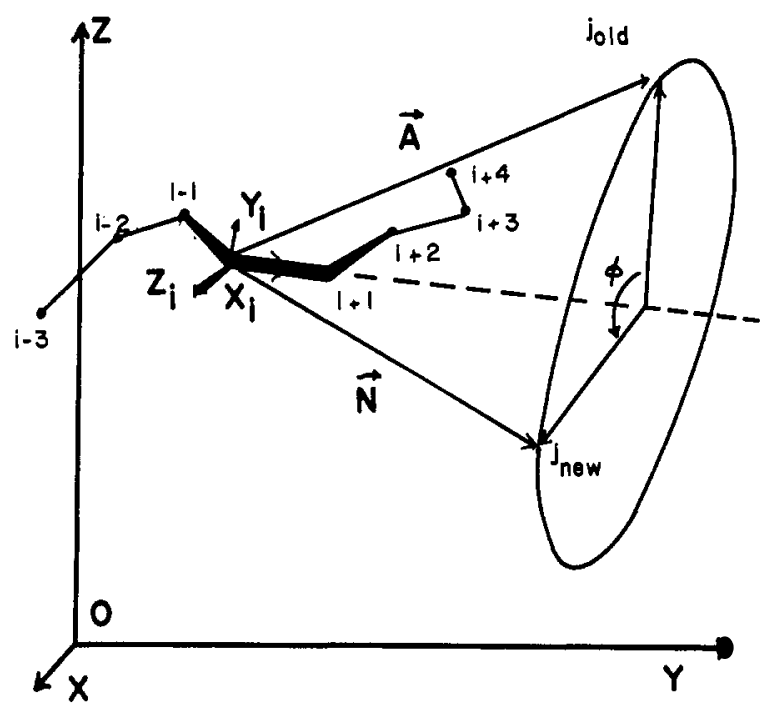

Figure 4. Segment of polymer molecule showing atoms $i-3$ through $i+4$, and atom $j>i+4$. Also shown is local coordinate system fixed on atom $i$, and its orientation with respect to the laboratory frame, 0 . Rotational transformation of eq 17 rotates atom $j$ through the angle $\phi$ about the extension of bond vector $x_{i}$ from its old position, $j_{\text {old }}$, to its new one, $j_{\text {new }}$.

where $r_{j k}$ is the distance between the $j$ th and $k$ th atoms, and the sum goes over all nonbonded pairs of atoms. The actual function, $f(r)$, is that of eq 4 , where $\rho$ was taken here to be $1.54 \AA$ or one $\mathrm{C}-\mathrm{C}$ single bond length. No side groups were introduced, and free rotation through $0-360^{\circ}$ was permitted about each single bond.

B. Mechanics of Simulation. The Monte Carlo experiment itself is carried out as described in the first section, namely, as a random walk in conformation space. This is done in principle as follows. Starting with any randomly chosen conformation $\omega$, a new one is reached by choosing at random a pivotal backbone atom $k, k \epsilon[1, n]$, and a rotational increment $\phi, \phi \in[0,2 \pi]$. This angle $\phi$ is then added modulo $2 \pi$ to the particular dihedral angle designated by the index $k, t i z$, $\omega_{k}$. In other words, if $\omega_{k}+\phi$ exceeds $2 \pi$, then $2 \pi$ is subtracted from the result. This rotates all backbone atoms numbered $k+1$ through $n$, inclusive, along with their attached side groups (if any), through circular arcs of angularity $\phi$ about the extension of bond $l_{k}$ connecting atoms $k$ and $k+$ 1 , as shown in Figure 4. By this means, a new point in conformation space is reached corresponding to a polymer which differs from the previous polymer in exactly one dihedral angle (viz., the $k$ th one), but which can be considerably different from it in overall shape and properties. The energy of this new conformation, $U$, is now computed along with any other desired properties, and stored in memory.

The reaching of a new point in conformation space is the computational analog of selecting a new random polymer from a real ensemble. In terms of the importance sampling scheme of Figure 2, this process corresponds to choice of conformation " $j . "$ It is a tentative choice, to be confirmed or rejected according to the biasing scheme.

If accepted, each rotation moves the representative point in conformation space a distance $\phi$ parallel to the $\omega_{k}$ axis. Successive rotations cause the point representing the accepted polymer (currently in machine memory) to execute a random walk in conformation space. The Markoffian nature of this walk is evidenced by the fact that each step obviously depends only on the immediately preceding position, and on no other. 
There are four main computational procedures to be described: (a) generation of initial coordinates, that is, the initial random $\omega$ from which the walk begins; (b) the relaxation transformation for rotation of the chain through $\phi ;$ (c) the corrector routine for improving the coordinates generated by (b); and (d) the algorithm for computing the energy of the new polymer. A fifth section (e) describes some utility programs developed for specific purposes.

(a) Generation of Initial Coordinates. We use the matrix equations of Scott and Scheraga ${ }^{26}$ for the construction of polymer coordinates satisfying a given geometry. The input to this scheme is the set of fixed bond lengths and bond angles, defined in Figure 3, and the dihedral angles $\omega_{i}$ as generated by eq 25 below. The initial conformation is totally random. The Scott-Scheraga algorithm is efficiently performed by judicious intermediate storage of the successively accumulated matrix products of the transformations. ${ }^{27}$

(b) Rotational Transform. The change of a single internal coordinate, $\omega_{k}$, leads to changes in a great number of Cartesian coordinates, three for every atom of index higher than $k$. In the program, if $k$ is less than $n / 2$, the front half of the chain is rotated rather than the rear half. This cuts the average number of atoms moved from about $3 n / 2$ to about $3 n / 4$, a considerable saving in computing time.

The formula for the forward rotation is as follows. Let $k$ be the index of a randomly selected pivotal atom, and consider the rotation of an atom with index $j, j>k$, from a position $j_{\text {old }}$ to a position $j_{\text {new }}$ as in Figure 4. Let $\mathbf{A}$ be the vector from atom $k$ to $j_{\text {old }}$ in the laboratory frame 0 , and $\mathbf{N}$ the vector from $k$ to $j_{\text {new }}$, also in the lab frame. Then

$$
\mathbf{N}=\mathbf{Q A}
$$

where

$$
\mathbf{Q}=\mathbf{D}^{-1} \mathbf{R}(\phi) \mathbf{D}
$$

and the orthogonal and unitary transformation matrix $\mathbf{R}(\phi)$ is given by

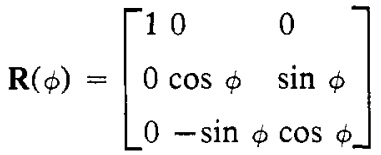

$\mathrm{D}$ is the matrix of direction cosines ${ }^{28}$ relating the local coordinate system fixed on atom $k$ back to the laboratory frame 0 . Although there are nine different direction cosines, they are not all independent and in reality only three need to be com-
Let $\mathbf{u}_{k}$ be the vector from the origin to the $k$ th atom. Then the new coordinates of atom $j$ after rotation are the components of a vector $\mathbf{u}_{j}^{\prime}$ given by

$$
\mathbf{u}_{j}{ }^{\prime}=\mathbf{u}_{k}+\mathbf{N}_{j}(j=k+1, \ldots, n)
$$

The simulation process corresponding to a single step of a random walk in conformation space then consists of choosing a random pivotal atom, $k$, a random angle, $\phi$, and a matrix $\mathbf{Q}$. The entire set of new coordinates for the affected chain segment is then given by application of eq 22, iterating on $j$.

(c) Correction for Machine-Induced Error. Up until now no explicit provision has been made for the finite capabilities of the computer itself. All the formulas derived so far can be assumed correct, in the context of purely mathematical abstraction. Unfortunately, all computers have physical limitations in their ability to evaluate such formulas.

One rather severe problem which was observed early in the course of this work was the tendency of the polymer molecule to shrink during the course of successive relaxations. This was traced to propagation of a certain type of roundoff error in the component manipulations of the relaxation arithmetic. A thorough investigation of this error disclosed an exponential propagation. For a chain of 92 atoms, the squared error in the bond length $l_{90}$ was found to obey

$$
\Delta_{90}{ }^{2}(k)=1.318 \times 10^{-9} \exp (0.08013 k)
$$

where $\Delta_{90}{ }^{2}$ is the difference between $l_{90}{ }^{2}$ and its supposedly true value of $(1.54)^{2}$, and $k$ is the number of times atom 90 was rotated by the previously described matrix transform. Typically, only about 400 such successive rotations were required to reduce the number of remaining significant digits in $l_{90}{ }^{2}$ from the original 14 to 2 . Since the sample size for a typical random walk in conformation space is of the order of $10^{4}$, which far exceeds the point at which coordinates produced by the above generating scheme lost all numerical significance, a self-correcting renormalization method was derived for correcting the coordinates as soon as they are generated. The mathematical details of this scheme are derived in the Appendix.

In the programs, the corrector equations are applied after each rotation, to every atom which has been moved, working from the pivotal atom outward. It was found that such an application added approximately $10 \%$ to the running time of the programs, but that now the successive rotations could be continued indefinitely without buildup of machine-induced error.

$$
\mathbf{Q}=\left[\begin{array}{lll}
a_{11}{ }^{2}+\left(1-a_{11}{ }^{2}\right) \cos \phi & a_{11} a_{12}(1-\cos \phi)+a_{13} \sin \phi & a_{11} a_{13}(1-\cos \phi)-a_{12} \sin \phi \\
a_{11} a_{12}(1-\cos \phi)-a_{13} \sin \phi & a_{12}{ }^{2}+\left(1-a_{12}{ }^{2}\right) \cos \phi & a_{12} a_{13}(1-\cos \phi)+a_{11} \sin \phi \\
a_{11} a_{13}(1-\cos \phi)+a_{12} \sin \phi & a_{12} a_{13}(1-\cos \phi)-a_{11} \sin \phi & a_{13}{ }^{2}+\left(1-a_{13}{ }^{2}\right) \cos \phi
\end{array}\right]
$$

puted. Multiplying out the elements of eq 18 , we find $\mathbf{Q}$ given by eq 20 , where $a_{11}, a_{12}$, and $a_{13}$ are the elements of any row of $\mathbf{D}$. Further simplification is possible by choosing

$$
\begin{array}{r}
a_{11}=\left(x_{k+1}-x_{k}\right) / l_{k+1} ; a_{12}=\left(y_{k+1}-y_{k}\right) / l_{k+1} ; \\
a_{13}=\left(z_{k+1}-z_{k}\right) / l_{k+1}
\end{array}
$$

in which the $x, y$, and $z$ coordinates are those of backbone atoms $k+1$ and $k$, referred to the laboratory frame 0 .

(27) This is illustrated in a flow chart in S. D. Stellman, Ph.D. Thesis, New York University, 1971.

(28) H. Margenau and G. M. Murphy, "The Mathematics of Physics and Chemistry," Vol. I, 2nd ed, Van Nostrand, Princeton, N. J. 1956, p 138 . (d) Energy-Computing Algorithms. A major time-consuming aspect of Monte Carlo polymer studies has always been the determination of the polymer energy. For simple self-avoiding walks, in which the energy is zero unless an overlap occurs, considerable ingenuity has been devoted to developing schemes for the detection of overlaps. Among these are the space-cubing method of Levinthal ${ }^{29}$ and the doubly linked list method of Gans. ${ }^{7}$ For a soft-core energy analogous procedures are possible, but can become quite tedious. In either case, however, if no precautions are taken computing time must rise in proportion to the square of the chain length.

(29) C. Levinthal, Sci, Amer, 214 (6), 42 (June, 1966) 
To cut down this dependence, an indexing method was developed for evaluating double sums such as occur in eq 16 . In our method, the index $i$ runs from 1 to $n-2$, as usual, but the index $j$ is permitted to skip whole sets of values which are excluded by geometric considerations. The details of this "zippering" method have been published by Stellman, Froimowitz, and Gans; ${ }^{30}$ for the hard-core energy model the time dependence was reduced to proportionality to $n^{8 / 4}$.

(e) Utility Programs. In addition to the major algebraic processes whose computational details were described above, four minor but essential subprograms were coded in machine language for speed.

(i) Random-Number Generator. At the heart of the Monte Carlo method must lie a fast method for supplying the program with a virtually unlimited sequence of random numbers, $\xi_{i}$. The properties which the $\xi_{i}$ 's must have are (i) they must be uncorrelated and (ii) they must be rectangularly distributed. The generator used in this work is the popular congruential pseudorandom-number generator, ${ }^{1}$ given by the recursive formula

$$
\xi_{i+1}=c \xi_{i} \operatorname{modulo}\left(2^{48}\right)
$$

where $c$ is a constant which produces no detectable correlation. In our program, an initial value, $\xi_{1}$, is read in, and is usually chosen to be the terminating $\xi_{\text {final }}$ of the preceding run. Occasionally, groups of 10,000 consecutively generated random numbers were tested for mean, variance, and rectangularity, to ensure validity of the results.

(ii) Random-Angle Generator. The random angle $\phi$ is not chosen directly, but its sine and cosine are generated by a method of von Neumann. ${ }^{31}$ In this method, two random numbers, $\xi_{1}$ and $\xi_{2}$, each uniformly and independently distributed in the open interval $(0,1)$, are generated, and the sum of squares, $\xi_{1}{ }^{2}+\xi_{2}{ }^{2}$, is formed. If this sum is greater than unity, the pair is rejected and $\xi_{1}$ and $\xi_{2}$ are chosen anew. Otherwise

$$
\cos \phi=\frac{\xi_{1}{ }^{2}-\xi_{2}{ }^{2}}{\xi_{1}{ }^{2}+\xi_{2}{ }^{2}} \quad \sin \phi=\frac{ \pm 2 \xi_{1}{ }^{2} \xi_{2}{ }^{2}}{\xi_{1}{ }^{2}+\xi_{2}{ }^{2}}
$$

The necessity of computing a third random number in order to choose at random the sign of the sine function in (25) has been obviated by assuming in the program that the 30th binary digit in the machine representation of $\xi_{1}$ is not correlated with the most significant digit, and using the former as a masking bit: if it is 1 , then the negative sign is taken, and if it is 0 , the positive sign is used. Angles generated in this way have satisfactorily passed various tests for randomness.

(iii) Random-Atom-Index Generator. Although there are $n$ internal coordinates $\omega_{i}$, actually three of them serve to fix the geometry of the entire polymer with respect to the laboratory frame. Hence, only $n-3$ are required to describe the internal geometry, and this is the number of angles which are varied in the program. Selection of a random pivotal atom $k$ is made by generating a random number $\chi$ in the open interval $(3, n)$, and truncating the result to an integer

$$
k=[\chi]
$$

(Square brackets denote the greatest integer not exceeding the given quantity.) The generation of a random number in this

(30) S. D. Stellman, M. Froimowitz, and P. J. Gans, J. Comput. Phys., 7, 178 (1971).

(31) D. von Neumann, Nat. Bur. Stand. Appl, Math. Ser., 12, 36 (1951). interval is achieved by generating $\xi_{i}$ according to eq 24 , multiplying it by $(n-3)$, and adding 3 to the result

$$
\begin{aligned}
\chi^{\prime} & =(n-3) \xi_{i} \epsilon(0, n-3) \\
\chi & =\chi^{\prime}+3 \epsilon(3, n) \\
k & =[\chi]=\left[\chi^{\prime}+3\right] \epsilon(3, n-1)
\end{aligned}
$$

as desired.

(iv) Solution of Linear Equations. In the coordinate improving routine (part c above), it is required to solve a system of three linear equations in three unknowns. This is done directly (noniteratively) by the method of Willers. ${ }^{32}$ The time required is one-half that of the normal Gaussian elimination method used in most linear systems, the saving resulting from performance of all computations in-line,

(f) Calculation of Properties. Properties are computed after each new acceptance directly from Cartesian coordinates currently in memory. For hard-core polymers, the properties of interest are the moments of the end-to-end distance and radius of gyration. These are computed as follows.

(i) Squared End-to-End Distance. This is found from

$$
R_{i}{ }^{2}=\left(x_{n}-x_{1}\right)^{2}+\left(y_{n}-y_{1}\right)^{2}+\left(z_{n}-z_{1}\right)^{2}
$$

where the subscript $i$ refers to the $i$ th accepted polymer in the data group and the other quantities are the $x, y$, and $z$ coordinates of the two end carbon atoms.

(ii) Squared Radius of Gyration. This is calculated as

$$
G_{i}^{2}=M^{-1} \sum_{k=1}^{n} m_{k} p_{k}^{2}-c^{2}
$$

where $M$ is the molecular weight of the polymer, $m_{k}$ is the atomic weight of the $k$ th atom, $p_{k}$ is the distance of the $k$ th atom from the origin, and $c$ is the distance of the center of gravity of the molecule from the origin.

Let $P_{i j}$ be the value of the property $P$ for the $i$ th polymer that was accepted in the $j$ th group, and $\eta_{i j}$ be the number of times it was accepted. Let $t$ be the number of different polymers accepted per group and $u$ be the number of groups. The total number of samples, $N$, is simply the sum of the degeneracies of all polymers in all groups

$$
N=\sum_{j=1}^{u} \sum_{i=1}^{t} \eta_{i j}
$$

The moments are computed as weighted averages of properties

$$
\left\langle P^{k}\right\rangle=P^{(k)}=N^{-1} \sum_{j=1}^{u} \sum_{i=1}^{t} \eta_{i j} P_{i j}^{k}
$$

in which $P_{i j}$ can be end-to-end distance, radius of gyration, or for soft-core calculations, the energy. For $R$ and $G$, the 1 st through 12 th moments were computed ( $k=1-12$ in eq 30$)$. To avoid the extreme magnitudes of the higher moments, reduced moments $\delta(p, s)$ were also calculated

$$
\begin{aligned}
& \delta_{R}(p, s)=\left\langle R^{p}\right\rangle /\left\langle R^{s}\right\rangle^{p / s} \\
& \delta_{G}(p, s)=\left\langle G^{p}\right\rangle /\left\langle G^{s}\right\rangle^{p / s}
\end{aligned}
$$

Histograms, or frequency counts of values falling within specified ranges, were made for $R$ and $G$ by sorting these values into bins as they were read from tape for the other calculations. Such data are equivalent to a numerical distribution function for the given property.

(32) A. Willers, "Practical Analysis," Dover Publications, New York, N. Y., 1948, pp 265-267. 
TABLE I

Results of Hard-Sphere Monte Carlo Calculations on $n$-Alkanes ${ }^{a}$

\begin{tabular}{rlrrrrrrr}
\hline$n$ & Run & \multicolumn{1}{c}{$N$} & \multicolumn{1}{c}{$f_{\mathrm{A}}$} & $t_{10 \mathrm{~K}}$ & $R^{2}$ & $\sigma\left(R^{2}\right)$ & $G^{2}$ & \multicolumn{1}{c}{$\sigma\left(G^{2}\right)$} \\
\hline 20 & II & 10,000 & 0.898 & 88.1 & 115.0388 & & 17.4982 & \\
20 & III & 10,000 & 0.899 & 137.8 & 114.1052 & 67.192 & 17.3621 & 5.3475 \\
30 & I & 10,000 & 0.874 & 166.3 & 202.1348 & & 30.4977 & \\
40 & I & 10,000 & 0.846 & 222.8 & 281.7629 & 171.1242 & 42.9679 & 14.5535 \\
40 & II & 10,000 & 0.853 & 286.0 & 288.1949 & 181.9059 & 44.1580 & 15.8454 \\
60 & I & 10,000 & 0.818 & 379.0 & 453.8205 & 290.4628 & 69.9711 & 26.1029 \\
60 & II & 10,000 & 0.822 & 379.6 & 468.4028 & 311.2770 & 71.6654 & 26.1618 \\
60 & III & 10,000 & 0.820 & 457.8 & 473.2606 & 307.0986 & 71.4499 & 27.3506 \\
80 & I & 10,000 & 0.802 & 577.8 & 674.7604 & 439.9251 & 104.3699 & 39.5151 \\
80 & II & 10,000 & 0.802 & 705.8 & 679.2795 & 446.5582 & 104.1964 & 39.7697 \\
98 & I & 10,000 & 0.793 & 909.2 & 856.4079 & & 129.4268 & \\
98 & II & 10,000 & 0.791 & 868.1 & 865.3902 & 582.5759 & 134.9050 & 53.0826 \\
298 & I & 4,000 & 0.735 & 3151.8 & 3460.213 & & 554.1066 & 481.8270 \\
298 & II & 4,700 & 0.716 & 2933.2 & 3031.795 & & 506.5307 & 534.6867
\end{tabular}

a $\left(\mathrm{C}_{n} \mathrm{H}_{2 n+2}\right) N$ is the number of samples per run, $f_{\mathrm{A}}$ is the fraction accepted, $t_{10 \mathrm{~K}}$ is the time in seconds per 10,000 samples (generation and analysis), and $\sigma$ is the standard deviation for the given data group.

TABLE II

Values of Fitted Fisher-Sykes Parameters for Various Lattices and Sampling Methods

\begin{tabular}{|c|c|c|c|c|c|}
\hline \multirow[b]{2}{*}{ Lattice type } & \multicolumn{4}{|c|}{ _-Direct (Wall) sampling _ _ _ _ } & \multirow{2}{*}{$\begin{array}{c}\text { Importance Sampling } \\
\text { This work }\end{array}$} \\
\hline & $\mathrm{SC}^{a}$ & $\mathrm{FCC}^{b}$ & Tet $^{c}$ & $\mathrm{Off}^{d}$ & \\
\hline$B$ & 1.00279 & 1.00926 & 0.94195 & & 1.1789 \\
\hline$C$ & 0.93728 & 0.91274 & 0.95953 & & 1.0001 \\
\hline$\alpha$ & 0.1459 & 0.1451 & 0.15457 & & -0.09014 \\
\hline$\lambda=-\ln C$ & 0.06477 & 0.09130 & 0.04131 & $0.059 \pm 0.001$ & $-1.0000 \times 10^{-4}$ \\
\hline
\end{tabular}

${ }^{a}$ Simple cubic; ref 5. ${ }^{b}$ Face-centered cubic; ref 5. ${ }^{c}$ Tetrahedral; ref 33. ${ }^{d}$ Reference 6.

The fraction of polymers accepted, $f_{\mathrm{A}}$, is the total number accepted divided by the total number generated.

\section{(II) Results of Calculations}

The hard-core studies were performed for three reasons: (1) to test the integrated operation of the various programs by attempting to duplicate some of the $\left\langle R^{2}\right\rangle$ and $\left\langle G^{2}\right\rangle$ data of Loftus and Gans ${ }^{6}$ (hereafter designated as LG); (2) to extend their data to $n=298$ in order to try to detect a deviation from their observed exponential dependence, if any; and (3) to obtain detailed information on the distribution of $R$ (or $R^{2}$ ). The hard-sphere parameter $\rho$ in eq 4 was taken as $1.54 \AA$, as in the LG studies. Hydrogen side groups were not considered, and free rotation was assumed about the dihedral angles. This study differs from LG only in the sampling technique, the latter having used the Wall method (Figure 1) with the Wall-Erpenbeck $s-p$ method of chain enrichment. ${ }^{20}$

The main results of this study are displayed in Table $I$, and include the population means and variances for $R^{2}$ and $G^{2}$, running times, fraction of samples accepted, and distributions (to be discussed in a future paper).

(1) Running Time. In addition to the usual molecular properties, attention must be paid to running time as a reflection of efficiency, because the more efficient the program, the greater the quantity of concrete results which can be obtained in a given allotment of computer time. As a practical matter, we have found ourselves so far limited to chain lengths below about 300 (and temperatures above $300^{\circ} \mathrm{K}$ in the soft-core studies).

Apart from the efficiency inherent in importance sampling, three types of programming improvements have been utilized to reduce running time. (1) Several subroutines were coded and optimized in machine language. (2) In certain cases more efficient algorithms were developed. For example, the generation of the initial chain was performed by successive matrix multiplications, with intermediate results stored in a particularly efficient way. The best example of algorithm improvement is the calculation of the energy by "zippering," described in a previous paper. ${ }^{30}$ In that paper, a shortcut for calculating the greatest integer in a square root is also given, a technique which saves $40 \%$ over the machine-supplied square root function. Thirdly, the $3 \times 3$ determinant for solving the linear equations generated in the coordinate-improving routine is performed in-line (no loops). (3) Inputoutput interaction with the program is kept to a minimum.

Comparison with other studies is not possible because almost no one else has published timing information, and because the CDC 6600 is the fastest computer applied to such studies so far. However, an agreeable demonstration of internal efficiency was made by plotting $\ln T v s . \ln n$, from which it was observed ${ }^{30}$ that the time required for these hard-core studies is proportional to $n^{5 / 4}$, that is

$$
T \propto n^{5 / 4}
$$

where $T$ is the time required for a given number of polymer generations and their analysis. By contrast, with no optimization $T$ ought to rise at least as fast as $n^{2}$.

(2) Fraction Accepted. It was pointed out above that for direct sampling, the partition function $Q$ is estimated by $f_{\mathrm{A}}$. This is true because in that method the fraction of polymers obtained which have overlaps (infinite energy) is in proportion to the volume of $\omega$ space which is forbidden. With importance sampling, however, polymers are deliberately generated more often in allowed regions and less of ten in forbidden re- 


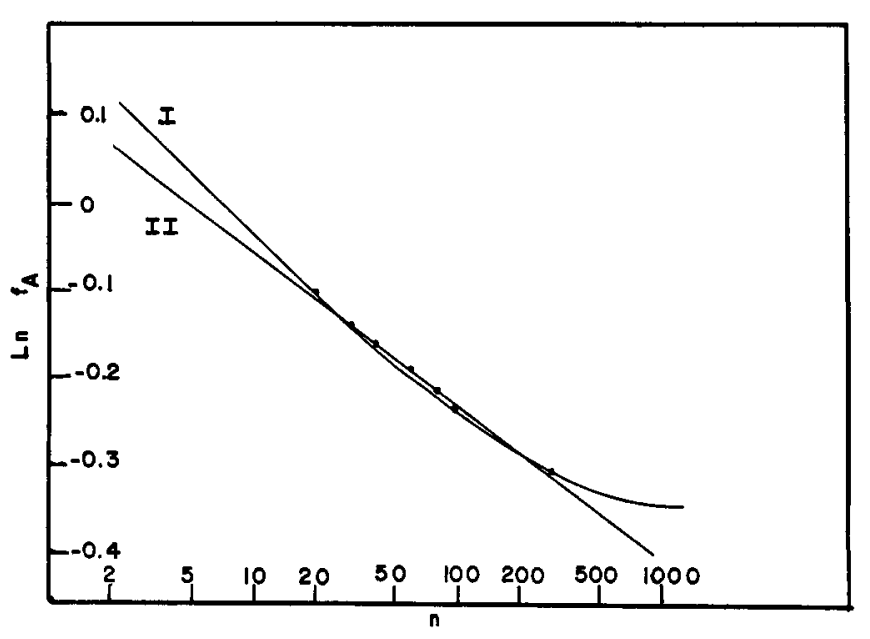

Figure 5. Log- $\log$ plot of $f_{\mathrm{A}}$ is. $n$ : curve I, eq 35 ; curve II, eq 36; circles, hard-sphere data.

TABLE III

Results of Least-Squares Fit of Mean-Squared End-to-End Distance and Radius of Gyration to EQ 37 and 38

\begin{tabular}{lrrrr} 
& \multicolumn{1}{c}{ Incl $n=20$} & Excl $n=20$ & Incl $n=20$ & Excl $n=20$ \\
\hline $\operatorname{Ln} a_{\bar{F}}$ & 1.0752 & 1.1544 & 0.8967 & 0.9067 \\
$b_{\mathrm{r}}$ & 1.2377 & 1.2209 & 1.2836 & 1.2812 \\
$\sigma\left(b_{\mathrm{r}}\right)$ & 0.0119 & 0.0054 & 0.0153 & 0.0346 \\
$\operatorname{Ln} a_{\mathrm{g}}$ & -0.8568 & -0.7920 & -0.9849 & -0.9674 \\
$b_{\mathrm{g}}$ & 1.2515 & 1.2378 & 1.2828 & 1.2787 \\
$\sigma\left(b_{\mathrm{g}}\right)$ & 0.0100 & 0.0056 & 0.0034 & 0.0070
\end{tabular}

${ }^{a}$ Reference 6.

gions, so that $f_{\mathrm{A}}$ is not an estimator of $Q$. In fact, when a single $\omega$ at a time is varied, as in these studies, $f_{\mathrm{A}}$ gives an estimate only of that fraction of forbidden hyperspace, which is at most one step away from an allowable region. Clearly, by underestimating the forbidden regions, one also underestimates the entire volume of space and therefore overestimates $Q$, that is

$$
f_{\mathrm{A}} \geqslant Q
$$

While the exact relationship of $f_{A}$ to $Q$ could not be guessed, it was obviously of interest to try to express $f_{A}$ in terms of a Fisher-Sykes ${ }^{18}$ type relation

$$
f_{\mathrm{A}}=B C^{n} n^{\alpha}
$$

where $B, C$, and $\alpha$ are constants to be determined. Using a simple linear least-squares fit, it was found that eq 34 gave an excellent fit to the data of Table I, with the parameters

$$
f_{\mathrm{A}}=1.1789(1.0001)^{n} n^{-0.09014}
$$

The sum of the squared residuals was $2.70 \times 10^{-5}$. These figures are compared in Table II with the values found by Gans $^{33}$ for the tetrahedral lattice, as well as by Mazur and McCrackin ${ }^{5}$ for the simple cubic and face-centered cubic lattices.

The value of 1.0001 for $C$, coupled with similar soft-core results, suggested that perhaps within error limits $C \equiv 1$. Therefore, $f_{A}$ was also fit to a simple exponential equation of the type $f_{\mathrm{A}}=a n^{b}$, with the result

$$
f_{\mathrm{A}}=1.1246 n^{-0.0758}
$$

(33) P. J. Gans, J. Chem. Phys, in press.

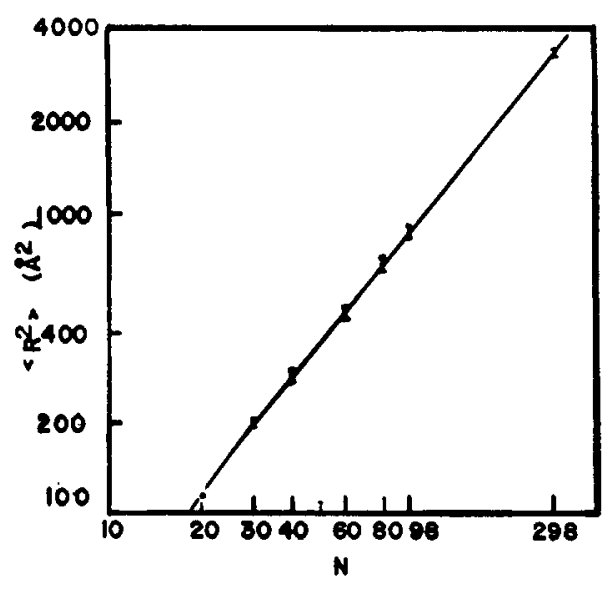

Figure 6. Log-log plot of $\left\langle R^{2}\right\rangle$ vs. $n$, with $90 \%$ confidence limits on the slope.

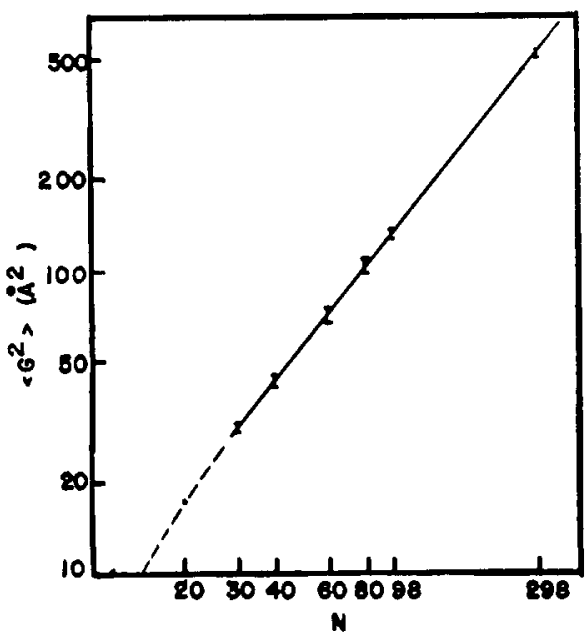

Figure 7. Log-log plot of $\left\langle G^{2}\right\rangle$ vs. $n$, with $90 \%$ confidence limits on the slope.

The standard deviation of the fitted exponent was 0.0025 . The plots of eq 35 and 36 are shown with actual data in Figure 5 .

It is interesting to note that if eq 35 holds, then $-\ln C$ has the meaning of an attrition constant, $\lambda$. In this context, our results demonstrate clearly that the importance sampling has effectively zero attrition; corresponding values of $\lambda$ for other sampling models and lattices are given in Table II, including the Loftus-Gans result 0.059 .

(3) Mean-Squared End-to-End Distance and Mean-Squared Radius of Gyration. Since the earliest Monte Carlo studies, it has been assumed that $\left\langle R^{2}\right\rangle$ and $\left\langle G^{2}\right\rangle$ obey the relationships

$$
\begin{aligned}
& \left\langle R^{2}\right\rangle=a_{\mathrm{r}} n^{b} \\
& \left\langle G^{2}\right\rangle=a_{\mathrm{g}} n_{\mathrm{g}}^{b}
\end{aligned}
$$

where $n$ is the number of segments (in $\mathrm{C}_{n} \mathrm{H}_{2 n+2}$ ), and $a_{\mathrm{r}}, b_{\mathrm{r}}, a_{\mathrm{g}}$, and $b_{\mathrm{g}}$ are parameters. The values of the fitted constants are compared with those of LG in Table III. Since the latter authors did not specify how they obtained their fits, we refit their published data (ref 6, Tables II and IV) so that the comparison would be valid. The parameters in Table III are given for fits containing all data points, and also for fits in which the $n=20$ datum was excluded (induction effect). These latter fits were justified in that their standard deviations were roughly half those of the complete data fits. In all cases, our exponents fall within the $90 \%$ confidence intervals of LG. Plots of $\left\langle R^{2}\right\rangle$ and $\left\langle G^{2}\right\rangle$ vs. $n$ are given in Figures 6 and 7, re- 
TABLE IV

Values of the Ratio $\theta(n)=\left\langle G^{2}\right\rangle_{n} /\left\langle R^{2}\right\rangle_{n}$

\begin{tabular}{rcc}
\hline$n$ & This work & $\mathrm{LG}^{a}$ \\
\hline 20 & 0.1521 & 0.1519 \\
30 & 0.1509 & \\
40 & 0.1529 & 0.1527 \\
60 & 0.1527 & 0.1522 \\
80 & 0.1540 & 0.1484 \\
98 & 0.1535 & \\
100 & & 0.1542 \\
298 & 0.1575 & \\
& Av 0.15337 & 0.15188
\end{tabular}

a Reference 6.

spectively. The solid lines in those figures are eq 37 and 38 fitted to our data. The ratios $\theta_{n}=\left\langle G^{2}\right\rangle_{n} /\left\langle R^{2}\right\rangle_{n}$ are given in Table IV, along with the LG values.

All of these comparisons serve to establish that our results are identical with those obtained with direct sampling. In addition, the consistent exponential dependence indicates persistence of eq 37 and 38 through $n=298$, which is three times the longest chain lenghth considered by LG.

Regression analysis was performed on the $\left\langle R^{2}\right\rangle$ and $\left\langle G^{2}\right\rangle$ data in order to (i) establish on a rigorous statistical basis the empirical "goodness" of eq 37 and 38 , and (ii) fix confidence limits about the two regression slopes, $b_{\mathrm{r}}$ and $b_{\mathrm{g}}$, to determine whether they include within their confidence intervals either the lattice value $8 / 5$, or the zero-order perturbation value of $4 / 3$.

The data of Table I, pooled at each data point, were subjected to regression on the line

$$
y=b x+a
$$

where $x=\ln n$ and $y=\ln \left\langle R^{2}\right\rangle$ or $\ln \left\langle G^{2}\right\rangle$. The point $n=$ 20 was excluded. Analysis of variance was performed according to the method given in detail by Brookes, et al. ${ }^{34}$

For $b_{\mathrm{r}}$, the exponent for the end-to-end distance, the standard error was found to be

$$
\text { S.E. }\left(b_{\mathrm{r}}\right)=5.4429 \times 10^{-3}
$$

The fitted slope can therefore be stated as $1.2209 \pm(5.4429$ $\left.\times 10^{-3}\right)(2.132)$, or

$$
b_{\mathbf{r}}=1.2209: \mathrm{CONF}_{90 \%}[1.2093,1.2325]
$$

where the numbers in brackets are the lower and upper $90 \%$ confidence limits, respectively, obtained by multiplying the standard error by 2.132, the value of Student's $t$ for four degrees of freedom at the two-sided $90 \%$ confidence level. ${ }^{34}$ Similar analysis of variance for $\left\langle G^{2}\right\rangle$ yielded the results

$$
\begin{gathered}
\text { S.E. }\left(b_{\mathrm{g}}\right)=5.5576 \times 10^{-3} \\
b_{\mathrm{g}}=1.2378: \mathrm{CONF}_{90 \%}[1.2259,1.2496]
\end{gathered}
$$

The important observations and inferences are as follows. The standard errors in $b_{\mathrm{r}}$ and $b_{\mathrm{g}}$ are nearly identical, leading us to infer that whatever reliability is attached to one also applies to the other. These errors have a magnitude of approximately $0.5 \%$. The two confidence intervals overlap, so that we might conclude as is of ten done that an empirical proportionality exists between $\left\langle G^{2}\right\rangle$ and $\left\langle R^{2}\right\rangle$. However, a better test of significance of this hypothesis would be to perform linear regression on the data of Table IV. The result of such

(34) J. Brookes, I. G. Betteley, and S. M. Loxston, "Mathematics and Statistics for Chemists," Wiley, New York, N. Y., 1966, Chapter 14. a test, performed on $\ln \theta_{n}$ vs. $\ln n$, produces a value of the statistic $F$ of 59.237. The corresponding value from tables of $F$ for $(1,5)$ degrees of freedom at $99 \%$ significance is 21.2 . This is an extremely important result, because it demonstrates that, at least for the range of data examined in this work, the difference in the empirical exponents $b_{\mathrm{g}}$ and $b_{\mathrm{r}}$ is statistically significant; in other words, the squared radius of gyration increases more rapidly with molecular weight than does the squared end-to-end distance.

This inequality was examined in one further way. Wall, Windwer, and Gans ${ }^{4}$ had reported values of $b_{\mathrm{g}}$ and $b_{\mathrm{r}}$ for six different values of their energy parameter $\xi$, and Loftus and Gans' observations covered six values of their angle $\beta$. In addition, Windwer ${ }^{35}$ reported $b_{\mathrm{g}}=1.32$ and $b_{\mathrm{r}}=1.29$ for his mixed lattice, and Wall and Erpenbeck ${ }^{20}$ obtained $b_{\mathrm{g}}=$ 1.195 and $b_{\mathrm{r}}=1.18$ on a tetrahedral lattice. Together, these constitute 14 independent observations on and off lattice. If the slight differences between $b_{\mathrm{r}}$ and $b_{\mathrm{g}}$ arose solely from the randomness of the Monte Carlo method, then on average we would expect $b_{\mathrm{r}}$ to exceed $b_{\mathrm{g}}$ in about half of the observations. Yet, in every observed case $b_{\mathrm{g}}>b_{\mathrm{r}}$. The odds against this are quite large.

We emphasize this seemingly minor point for two reasons. First, even a tiny difference, appearing as it does in an exponent, can greatly affect predictions of polymer dimensions. Secondly, many authors ${ }^{36}$ base further results on the assumption of equality. Furthermore, the common practice of reporting end-to-end distances as simple multiples of experimentally observed radii of gyration is clearly incorrect.

Finally, we observe that neither the $b_{\mathrm{r}}$ or $b_{\mathrm{g}}$ confidence limits include either $6 / 5$ or $4 / 3$ at the $90 \%$ level. In other words, a straight line with the lattice slope of 1.2000 cannot be made to lie within the error envelope calculated for Figures 6 and 7. This confirms the observations of LG and of Windwer $^{35}$ that off-lattice statistics are significantly different from lattice values.

(4) Distribution Functions. The number of polymers, $f(R)$, at each chain length $n$ whose end-to-end distance $R$ fell within certain specified ranges was also noted.

These empirical distributions were fit to the expression

$$
f(R)=\exp \left[-\left(a R^{2}+b R+c\right)\right]
$$

By means of $\chi^{2}$ tests, the degree of fit to this two-parameter function was found to be statistically significant at the $95 \%$ confidence level, for $n=80$ and 98 , and at the $80 \%$ level for $n=298$ (lesser degree of fit possibly due to limitations of sample size). This equation, with parameters fit to the overall distribution, accurately reproduced the 1 st through 12 th independently observed moments of the distributions. A detailed discussion of further distribution function results and their implications in excluded volume theory is deferred to a future paper.

\section{(III) Summary}

The conformational properties of single $n$-alkane molecules containing from 20 to 298 carbon atoms were calculated by a Monte Carlo method, using a free-rotation hard-core potential energy model. The conformation space of an alkane was defined as the $m$-dimensional finite (bounded) space whose mutually orthogonal axes correspond to the molecular dihedral angles $\omega$ which are capable of internal rotation. The

(35) S. Windwer, J. Chem. Phys. 43, 115 (1965)

(36) For example Kumar and $S$ Windwer, ibid 47, 4057 (1968); F. T. Wall and F. T. Hioe, J. Phys. Chem., 74, 4416 (1970). The latter authors explicitly assume $b_{\mathrm{r}}=b_{\mathrm{g}}$ on the basis of enumeration of walks of fewer than 20 steps. 


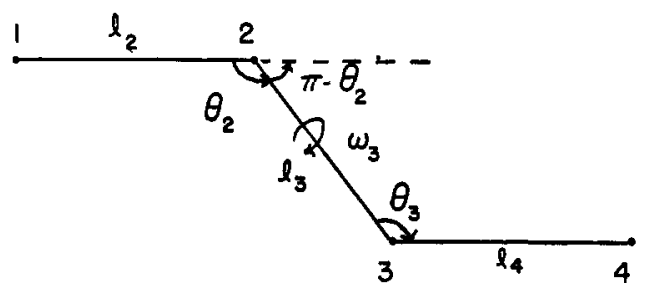

Figure 8. Definitions of bond lengths, bond angles, and dihedral angles for Appendix.

conformational partition function $Q$ was defined in eq 2 in terms of the $\omega$ 's, and the statistical-mechanical average value of a property, $\langle P\rangle$, was defined in eq 1 . It was shown that all lattice models of polymers fall into this broader classification in terms of $\omega$ space, and that the well-known Wall attrition law, eq 6, expresses the fractional volume of this space available to a particular molecular species.

In the first section, the mathematical principles underlying the Monte Carlo method were summarized. The actual application of these methods to the estimation of $Q$ and other properties was described in statistical terms. It was shown that the Monte Carlo integration required for the partition function was equivalent to a Markoffian random walk in $\omega$ space, with a weighting function equal to the Boltzmann factor. Once the correspondence between statistical mechanics and pure statistics was shown, a prebiasing scheme (importance sampling) was introduced to make the sampling more efficient.

The geometric model and computational details were described in section I, including equations for obtaining statistical-meclianical average properties from the data, and efficiency-improving techniques.

The hard-core results showed that the importance sampling method is equivalent to the Wall method (direct sampling), but that importance sampling has effectively zero attrition compared to the Wall scheme. The exponential dependence of $\left\langle R^{2}\right\rangle$ and $\left\langle G^{2}\right\rangle$ on $n$, the chain length, was found to be valid off-lattice at least up to $n=298$. Careful regression analysis revealed the new conclusion that the squared radius of gyration increases more rapidly at the chain lengths investigated than does the squared end-to-end distance. Furthermore, the $90 \%$ confidence limits calculated for both the $\left\langle R^{2}\right\rangle$ and $\left\langle G^{2}\right\rangle$ exponents did not include either $6 / 5$ or $4 / 3$, the lattice and zero-order perturbation values, respectively. This is a clear demonstration that lattice results, no matter how reliably obtained, may not be indiscriminately applied to real (nonlattice) polymers.

Acknowledgments. We wish to thank the AEC Computing and Applied Mathematics Center of the Courant Institute of Mathematical Sciences at New York University for a generous grant of computer time. Additional computer time was purchased through NSF Grant No. NSF-GU-1147, One of us (S. D. S.) is grateful to the National Science Foundation for a Graduate Traineeship.

\section{Appendix. Self-Correcting Scheme for Generating New} Coordinates by Successive Orthogonal Transformations

There are three coordinates to be generated for each atom undergoing relaxation, and there are three metrics which must be preserved during its rotation: bond length, bond angle, and dihedral angle. Let these quantities be defined as in Figure 8. We assume the coordinates of atoms 1, 2, and 3 to be without error (or may define their true values to be equal to their machine representations). Then the coordinates of the fourth atom (about to be added) are functions of the nine previous Cartesian coordinates. But in view of the additional metric constraints, we may write

$$
\begin{aligned}
& x_{4}=f_{1}\left(x_{3}, y_{3}, z_{3}, \theta_{3}, \omega_{3}\right)=g_{1}\left(l_{4}^{2}, \theta_{3}, \omega_{3}\right) \\
& y_{4}=f_{2}\left(x_{3}, y_{3}, z_{3}, \theta_{3}, \omega_{3}\right)=g_{2}\left(l_{4}^{2}, \theta_{3}, \omega_{3}\right) \\
& z_{4}=f_{3}\left(x_{3}, y_{3}, z_{3}, \theta_{3}, \omega_{3}\right)=g_{3}\left(l_{4}^{2}, \theta_{3}, \omega_{3}\right)
\end{aligned}
$$

These metrics, $\theta_{3}, \omega_{3}$, and $l_{4}{ }^{2}$ are related to the new coordinates $x_{4}, y_{4}$, and $z_{4}$ through the differentials

$$
\begin{array}{r}
\mathrm{d}\left(\cos \theta_{3}\right)=\frac{\partial\left(\cos \theta_{3}\right)}{\partial x_{4}} \mathrm{~d} x_{4}+\frac{\partial\left(\cos \theta_{3}\right)}{\partial y_{4}} \mathrm{~d} y_{4}+\frac{\partial\left(\cos \theta_{3}\right)}{\partial z_{4}} \mathrm{~d} z_{4} \\
\mathrm{~d}\left(\cos \omega_{3}\right)=\frac{\partial\left(\cos \omega_{3}\right)}{\partial x_{4}} \mathrm{~d} x_{4}+\frac{\partial\left(\cos \omega_{3}\right)}{\partial y_{4}} \mathrm{~d} y_{4}+\frac{\partial\left(\cos \omega_{3}\right)}{\partial z_{4}} \mathrm{~d} z_{4} \\
\mathrm{~d}\left(l_{4}{ }^{2}\right)=\frac{\partial\left(l_{4}{ }^{2}\right)}{\partial x_{4}} \mathrm{~d} x_{4}+\frac{\partial\left(l_{4}{ }^{2}\right)}{\partial y_{4}} \mathrm{~d} y_{4}+\frac{\partial\left(l_{4}{ }^{2}\right)}{\partial z_{4}} \mathrm{~d} z_{4} \text { (A-1) }
\end{array}
$$

The quantities appearing as differentials on the left-hand side of eq A-1 are to be taken in the sense of finite differences. For example, $\mathrm{dl} l_{4}{ }^{2} \sim \Delta_{4}{ }^{2}$, the true squared bond length minus the value computed from the error-prone coordinates of atom 4 as generated in the computer by eq 17-19. In a similar fashion, the differentials $\mathrm{d} x_{4}, \mathrm{~d} y_{4}$, and $\mathrm{d} z_{4}$ are approximated by $\Delta x_{4}, \Delta y_{4}$, and $\Delta z_{4}$, the respective differences between the true (but as yet unknown) $x_{4}, y_{4}$, and $z_{4}$ coordinates, and those computed by the machine.

The values of $l_{4}{ }^{2}, \cos \theta_{3}$, and $\cos \omega_{3}$ are first computed from the current coordinates 1,2 , and 3 , and the computed coordinates of atom 4 , taken temporarily to be correct. The designated partial derivatives are then evaluated, and the resulting equations are finally solved simultaneously for the unknowns, $\Delta x_{4}, \Delta y_{4}$, and $\Delta z_{4}$. These are the increments to be added algebraically to the coordinates of atom 4 produced by the rotational transformation in order to obtain their correct values.

The metric quantities are most easily evaluated by use of vector notation. Let the bond vectors be represented by $\mathbf{v}_{\mathrm{a}}, \mathbf{v}_{\mathrm{b}}$, and $\mathbf{v}_{\mathrm{c}}$

$$
\begin{aligned}
& \mathbf{v}_{\mathrm{a}}=\left(x_{2}-x_{1}, y_{2}-y_{1}, z_{2}-z_{1}\right) \\
& \mathbf{v}_{\mathrm{b}}=\left(x_{3}-x_{2}, y_{3}-y_{2}, z_{3}-z_{2}\right) \\
& \mathbf{v}_{\mathrm{c}}=\left(x_{4}-x_{3}, y_{4}-y_{3}, z_{4}-z_{3}\right)
\end{aligned}
$$

Then $l_{4}{ }^{2}, \cos \theta_{3}$, and $\cos \omega_{3}$ may be expressed in terms of the coordinates of atoms $1,2,3$, and (uncorrected) 4 as

$$
l_{4}^{2}=\mathbf{v}_{\mathrm{c}} \cdot \mathrm{v}_{\mathrm{c}}=\left(x_{4}-x_{3}\right)^{2}+\left(y_{4}-y_{3}\right)^{2}+\left(z_{4}-z_{3}\right)^{2}
$$

$\cos \theta_{3}=\mathbf{v}_{\mathrm{b}} \cdot \mathbf{v}_{\mathrm{c}}=-$

$$
\frac{\left(x_{3}-x_{2}\right)\left(x_{4}-x_{3}\right)+\left(y_{3}-y_{2}\right)\left(y_{4}-y_{3}\right)+\left(z_{3}-z_{2}\right)\left(z_{4}-z_{3}\right)}{l_{3} l_{4}}
$$

$\cos \omega_{3}=\frac{\left(\mathbf{v}_{\mathrm{a}} \times \mathbf{v}_{\mathrm{b}}\right) \cdot\left(\mathbf{v}_{\mathrm{b}} \times \mathbf{v}_{\mathrm{c}}\right)}{l_{2} l_{3}{ }^{2} l_{4} \sin \theta_{2} \sin \theta_{3}}=\frac{\cos \theta_{2} \cos \theta_{3}}{\sin \theta_{2} \sin \theta_{3}}-\frac{\mathbf{v}_{\mathrm{a}} \cdot \mathbf{v}_{\mathrm{c}}}{l_{2} l_{4} \sin \theta_{2} \sin \theta_{3}}$

For tetrahedral bond angles, the third of eq A-2 reduces to

$$
\left.\cos \omega_{3}\right|_{\theta_{2}=\tau}=\frac{1}{8}\left[1-9\left(\frac{\mathbf{v}_{\mathrm{a}} \cdot \mathbf{v}_{\mathrm{c}}}{l_{2} l_{4}}\right)\right]
$$


After evaluating the partial derivatives and substituting the assumed correct values of coordinates of atom 4 , we obtain

$$
\begin{aligned}
& \Delta l_{4}{ }^{2}= 2\left(x_{4}-x_{3}\right) \Delta x_{4}+2\left(y_{4}-y_{3}\right) \Delta y_{4}+2\left(z_{4}-z_{3}\right) \Delta z_{4} \\
& \Delta\left(\cos \theta_{3}\right)=\frac{\left(x_{2}-x_{3}\right)}{l_{3} l_{4}} \Delta x_{4}+\frac{\left(y_{2}-y_{3}\right)}{l_{3} l_{4}} \Delta y_{4}+\frac{\left(z_{2}-z_{3}\right)}{l_{3} l_{4}} \Delta z_{4} \\
& \Delta\left(\cos \omega_{3}\right)=\frac{9}{8} \frac{\left(x_{1}-x_{2}\right)}{l_{2} l_{4}} \Delta x_{4}+\frac{9}{8} \frac{\left(y_{1}-y_{2}\right)}{l_{2} l_{4}} \Delta y_{4}+ \\
& \frac{9}{8} \frac{\left(z_{1}-z_{2}\right)}{l_{2} l_{4}} \Delta z_{4}
\end{aligned}
$$

where $\Delta()$ denotes the difference between the known metric and its value computed from coordinates so far generated. This constitutes a system of three linear equations in three unknowns, viz., $\Delta x_{4}, \Delta y_{4}$, and $\Delta z_{4}$, which may be solved by any convenient method. ${ }^{32}$

The final step, after solution of eq A-3, is thus to form the corrected coordinates

$$
\begin{aligned}
& x_{4_{\mathrm{corr}}}=x_{4_{\mathrm{comp}}}+\Delta x_{4} \\
& y_{4_{\mathrm{corr}}}=y_{4_{\mathrm{comp}}}+\Delta y_{4} \\
& z_{4_{\mathrm{corr}}}=z_{4_{\mathrm{comp}}}+\Delta z_{4}
\end{aligned}
$$

\title{
${ }^{13} \mathrm{C}$ Nuclear Magnetic Resonance Spectra of $m$-Diethynylbenzene Polymers and Related Polyacetylenes ${ }^{1 \mathrm{a}}$
}

\section{Dwain M. White* and George C. Levy}

General Electric Research and Development Center, Schenectady, New York 12301. Received November 11,197/1

\begin{abstract}
Pulsed Fourier transform (FT) ${ }^{13} \mathrm{C}$ nmr has been used to analyze low molecular weight trimethylsilyl-terminated polymers from the oxidative coupling of $m$-diethynylbenzene and also a number of model arylacetylenes. The FT spectra have sufficiently high resolution and signal-to-noise ratios to measure resonances for nuclei in the end groups and to rule out the occurrence of extensive side reactions during polymerization. Other analyses, including ${ }^{1} \mathrm{H} \mathrm{nmr}$ and infrared spectroscopy support these findings.
\end{abstract}

$\mathrm{N}^{\mathrm{a}}$ atural-abundance ${ }^{13} \mathrm{C}$ nuclear magnetic resonance has been shown to be a very powerful tool in the investigation of polymer microstructure. ${ }^{2}$ The newly developing methods of pulsed Fourier transform (FT) ${ }^{13} \mathrm{C} \mathrm{nmr}$ do much to overcome the well-known sensitivity limitations of naturalabundance carbon $\mathrm{nmr}$ studies. ${ }^{2 \mathrm{~b}}{ }^{13} \mathrm{C}$ FT $\mathrm{nmr}$ is particularly suited for the andysis of the polymer II from the oxidative coupling of $m$-diethynylbenzene (I) (Reaction 1 ). Since ${ }^{13} \mathrm{C}$

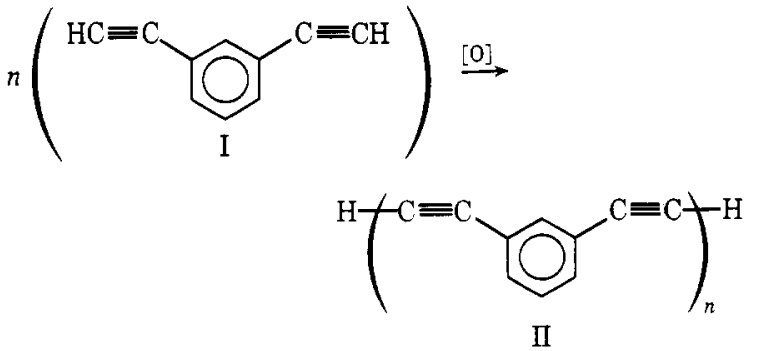

chemical shifts occur over a wide range ( $200 \mathrm{ppm})$, it should be possible to resolve nonequivalent carbons both in the aryl and acetylenic portions. With ${ }^{1} \mathrm{H} \mathrm{nmr}$, very little information is gained because of the relatively small number of protons and their similar chemical shifts. Utilization of Fourier ${ }^{13} \mathrm{C} \mathrm{nmr}$ may allow detection of the end groups and uncover the presence of any abnormal groupings. To achieve similar ${ }^{13} \mathrm{C}$ spectra by the usual frequency sweep nmr methods (continuous wave, $\mathrm{CW}$ ) would require experimental measurement

(1) (a) Part III, Polymerization by Oxidative Coupling; part II, D. M. White, J, Polym. Sci., Part A-1, 9, 663 (1971); (b) the publication of this paper was delayed owing to production difficulties.

(2) (a) J. Schaefer, Macromolecules, 2, 210 (1969); L. F. Johnson, F. Heatley, and F. A. Bovey, ibid., 3, 175 (1970); M. W. Duch and D. M. Grant, ibid., 3, 165 (1970); C. J. Carman, A. R. Tarpley, and J. H. Goldstein, ibid., 4, 445 (1971); (b) J. Schaefer, ibid., 4, 98, 105 , 110 (1971). times that exceed the sample's stability at the temperatures required for polymer dissolution. Another advantage is the high-resolution that can be attained, since ${ }^{13} \mathrm{C}$ spectra from $\mathrm{FT}$ experiments are not subject to the line broadening resulting from rapid sweeps in $\mathrm{CW} \mathrm{nmr}$.

The copper-amine-catalyzed oxidative coupling of $\mathrm{m}$ diethynylbenzene (reaction 1 ) was reported by Hay. ${ }^{3}$ Characterization by infrared spectroscopy, X-ray diffraction, and thermal analyses indicated that structure II was consistent with the properties of the polymer: ${ }^{4}$ however, limited solubility prevented detailed characterization of the polymer. Recently, methods of polymerization have been found which permit polymerization under homogeneous conditions. ${ }^{5}$ With these procedures, phenylethynyl-terminated polymers have been prepared with sufficient solubility to be characterized further. ${ }^{5}$ The polymers, however, were either copolymers of $m$ - and $p$-diethynylbenzene or terpolymers containing an additional diacetylene. The presence of repeating units other than the meta units tended to complicate the nmr spectra. Thus, a soluble all-meta polymer was desirable for a detailed examination by $\mathrm{nmr}$.

This paper describes polymers with $>99 \% \mathrm{~m}$-diethynylenephenylene backbone units which are terminated with trimethylsilyl groups. The polymers are particularly suited for $\mathrm{nmr}$ analysis both by ${ }^{1} \mathrm{H} \mathrm{nmr}$ (nine protons per trimethylsilyl group for end group analysis) and ${ }^{13} \mathrm{C} \mathrm{nmr}$, since the quantity of the monofunctional trimethylsilyl component can be varied to control the molecular weight. This permits the preparation of low molecular weight, more soluble polymers with high end group concentrations.

(3) A. S. Hay, J. Org. Chem., 25, 1275 (1960).

(4) A. E. Newkirk, A. S. Hay, and R. S. McDonald, J. Poll'm. Sci., Part A, 2, 2217 (1964).

(5) D. M. White, Polym. Prepr., Amer. Chem. Soc, Dir. Polym. Chem, 12 (1), 155(1971). 